\title{
Dynamic segmentation of aluminum wheel images based on rotation histogram
}

\author{
Shuhong Cheng ${ }^{1,2, a}$, Bin Zhou ${ }^{1, b}$, Shuchun Cheng ${ }^{3}$, Shijun Zhang ${ }^{1}$ \\ ${ }^{1}$ School of Electrical Engineering, YanShan University, China \\ ${ }^{2} \mathrm{CITIC}$ Dicastal Co.,Ltd Engineering Technology Institute, China \\ ${ }^{3}$ The first mine of the third Oil Plant, Daqing oil field, China \\ ashhcheng@ysu.edu.cn, b3114884526@qq.com
}

Keywords: image processing; aluminum wheel; rotating projection; dynamic segmentation.

\begin{abstract}
In order to solve the problem which about the information traceability in the field of automobile wheel, a method of dynamic segment the aluminum wheel images based on rotation histogram is proposed in this paper. And in this way, we can extract the information region from the back cavity of aluminum wheel. A segmentation method of ROI is put forward which based on histogram of 360 degree rotation projection. First, the image is rotated 360 degrees, then drawing the rotation histogram with the rotate of image, and the last, the region of interest is divided according to the projection histogram in a dynamic way. Experiments show that the proposed method can meet the circle characteristic of aluminum wheel and segment the ROI. Using this method can simplify the procedure of image processing, accelerate the progress of the process, and segment the effective regions farthest.
\end{abstract}

\section{Introduction}

Today, with the booming development of the automotive industry, a variety of accidents caused by the wheel of the car is also an endless stream. Therefore, the safety of production and information of the automobile wheel has become an urgent problem to be solved. It is a good way to use the image processing technology for the collected aluminum wheel images to extract and identify the information from the back cavity of aluminum wheel. However, one of the important contents of image processing is the effective segmentation of the image, which can quickly and accurately segment the effective area to finish the later stage of information identification. In this paper, the method of 360 degrees rotation projection histogram is proposed, according to the characteristics of circular, the rotating projection can reflect the information of the wheel farthest in the projection histogram and use this histogram can segment the ROI better. Researchers in the field of rotation projection also put forward different methods of application according to the direction of their research ${ }^{[1-3]}$. Besides,L.Liu et al analyse the rotation motion of circular feature based on the principle of perspective, and the method of calculating the rotation angle had been proposed. The measurement precision error of the rotation angle was increased to 0.4 degrees ${ }^{[4]}$. Peter D. Wentzell et al use the method of Procrustes analysis as a diagnostic tool, through establishing Comparative relationship between the rotation projection and the map of Procrustes analysis to track the soybean disease ${ }^{[5]}$. Bohong Wei et al. Apply the method of rotating projection to convert the two-dimensional image patches into one dimensional vector, and the template matching was performed according to the rotation invariant similarity measure ${ }^{[6]}$. Min Yang et al apply the rotational projection into the diagnosis of micro scanning $\mathrm{CT}^{[7]}$. Meng Dong et al use genetic programming and rotationally invariant local binary patterns to segment and process the image of human ovary, and its correct rate research $93.33 \%{ }^{[8]}$. For different application background, the researchers use the method of rotation to make it more effective for their own use, for example in the field of Biochemistry, Medical Science and geology, etc ${ }^{[9-14]}$. But all the methods are mentioned above just have the biggest pertinence in the field of their stydies. And Most of these methods fall into two categories: based on they can't rotate and based on they are rotated by camera. However, our 
identification of wheel of the camera can not rotate and we can't obtain the whole information of wheel if we can't use the rotation. So, what the rotation method that they said is different from mine and their methods can't finish my work in a better way. In this paper, we propose a rotation projection method that who use the rotation projection aimed at the wheel of the information in the most comprehensive way on the histogram, so that we can segment the ROI more easily.

It is special design application method that we propose in the paper which according to the feature of aluminum wheel, and experimental proof that the method of 360 degrees rotation projection histogram can be used in the back cavity of aluminum wheel, and it can achieve good results.

\section{Methods and Principles}

\subsection{Pretreatment}

In this study, we use the series of FJ camera to collect the pictures of the back cavity of aluminum wheel in the pretreatment process, as shown in Figure 1(a).First of all, we should finish the gray scale processing and filtering process, and then extracting the initial effective area of the image, as shown in Figure 1(b). To find and draw the outline of the circle in the circle of back cavity of preliminary extraction, according to the different sizes of the wheels to find out the inner circle contour and outer circle contour, as shown in Figure 1(c). When we find the two main circle contour, we should remove the region of the inner circle and only to retaining the final annular region, as shown in Figure 1(d), and all the important information of aluminum wheels are concentrated in the annular region.

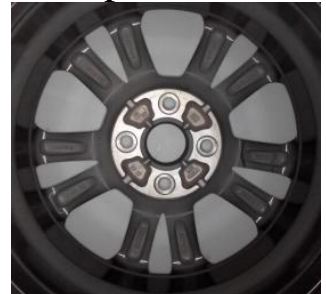

(a)Original image

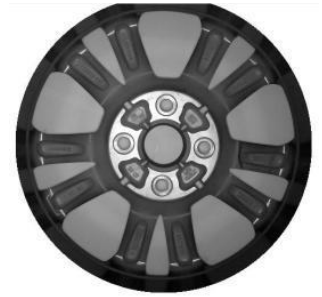

(b)Pre-extraction

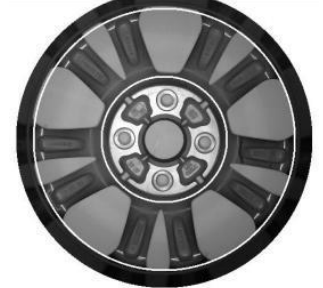

(c)Circular contour record

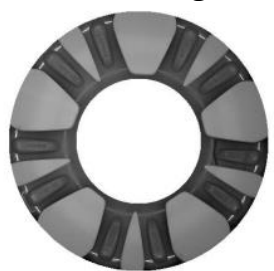

(d)Annular region image

Fig.1 Pretreatment diagram

\subsection{Image segmentation based on 360 degree rotation projection histogram}

\subsubsection{General image projection}

General image projection is the transverse projections or vertical projection. They are formed by traversing pixels from different directions.But this method can't complete the task that express the information of wheel image, and it also can't provide useful information for the image segmentation. As shown in Figure 2, the Figure 2(c) is the transverse projection histogram and Figure 2(d) is the vertical projection histogram. From these two pictures we can see that there are no useful information here. So, in order to obtain more useful information from the wheel image, we must make a chance in the aspect of projection method.

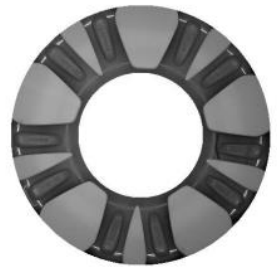

(a)Circular area image

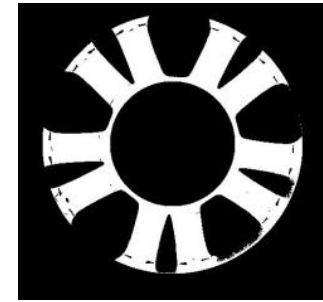

(b)Binarization

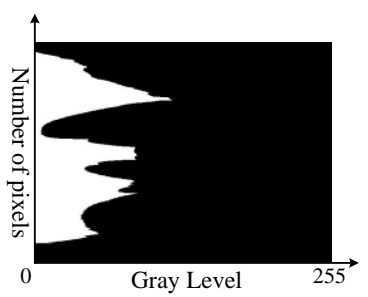

(c)transverse projection

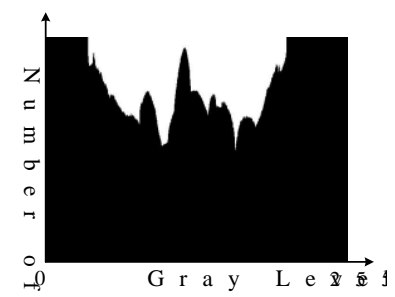

(d)vertical projection

Fig. 2 General image projection

\subsubsection{Image rotation projection}

The image rotation must determine the center point of its rotation around and the situation of rotation angle that is converted. As shown in Figure 3, the point $\left(x_{0}, y_{0}\right)$ is become the point $\left(x_{1}, y_{1}\right)$ after the rotary conversion. Its rotation transformation formula is expressed by formula (1) and formula (2). 


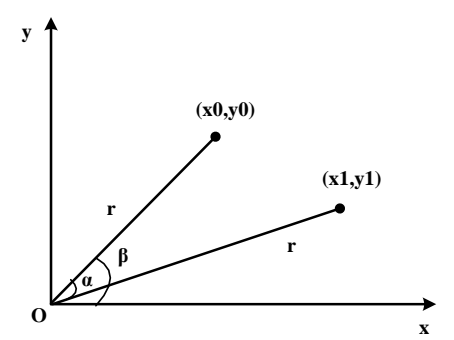

Before rotation:

Fig.3 Schematic diagram of image rotation

$\left\{\begin{array}{l}x_{0}=r \cos \beta \\ y_{0}=r \sin \beta\end{array}\right.$

After rotation:

$\left\{\begin{array}{l}x_{1}=r \cos (\beta-\alpha)=r \cos \beta \cos \alpha+r \sin \beta \sin \alpha=x_{0} \cos \alpha+y_{0} \sin \alpha \\ y_{1}=r \sin (\beta-\alpha)=r \sin \beta \cos \alpha-r \cos \beta \sin \alpha=-x_{0} \sin \alpha+y_{0} \cos \alpha\end{array}\right.$

According to the theory above, the rotation is done in a conventional XOY coordinate system, so the center of rotation is the origin $(0,0)$. But in the processing of image the coordinates system is locating in the fourth quadrant, and the origin is the upper left corner, rather than at the center point of the image, therefore the center point of origin image need to be transferred to the position that set by myself. As shown in Figure 4.

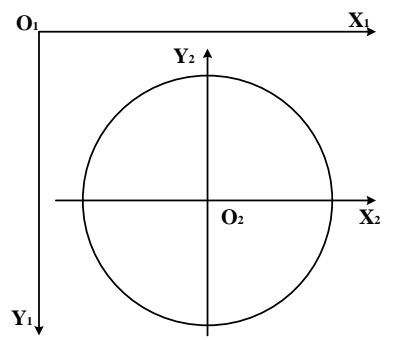

Fig.4 Schematic diagram of rotation center transformation

As shown above, the $\mathrm{X}_{1} \mathrm{O}_{1} \mathrm{Y}_{1}$ is the coordinate system of the image pixel traverse scanning, and the $\mathrm{X}_{2} \mathrm{O}_{2} \mathrm{Y}_{2}$ is the image rotation coordinate system, and its rotation center is the $\mathrm{O}_{2}$ point which is set up by myself. Therefore, it is need for us to realize the conversion between the two coordinate systems, and the rotation center of the processed image is $(\mathrm{m}, \mathrm{n})$, then the formula of the coordinate system transformation is represented by the formula (3) and the formula (4): transform matrix:

$$
\left[\begin{array}{l}
X_{2} \\
Y_{2} \\
1
\end{array}\right]=\left[\begin{array}{ccc}
1 & 0 & -m \\
0 & -1 & n \\
0 & 0 & 1
\end{array}\right]\left[\begin{array}{c}
X_{1} \\
Y_{1} \\
1
\end{array}\right]
$$

Inverse transformation matrix:

$$
\left[\begin{array}{c}
X_{1} \\
Y_{1} \\
1
\end{array}\right]=\left[\begin{array}{lll}
1 & 0 & m \\
0 & -1 & n \\
0 & 0 & 1
\end{array}\right]\left[\begin{array}{c}
X_{2} \\
Y_{2} \\
1
\end{array}\right]
$$

According to the formula (3), we can convert the coordinate system $\mathrm{O}_{1}$ into coordinate system $\mathrm{O}_{2}$, according to the formula (4), we can convert the coordinate system $\mathrm{O}_{2}$ into coordinate system $\mathrm{O}_{1}$. Assuming that in the new coordinate system, we define that the coordinate of the left upper corner of new image as the origin, the center coordinate of the image that not be rotated as $(a, b)$, and the center coordinate of the image that is rotated as $(\mathrm{c}, \mathrm{d})$, then the pixel point of the rotation transformation matrix is shown as formula (5): 


$$
\left[\begin{array}{r}
x_{1} \\
y_{1} \\
1
\end{array}\right]=\left[\begin{array}{lll}
1 & 0 & c \\
0 & -1 & d \\
0 & 0 & 1
\end{array}\right]\left[\begin{array}{lll}
\cos \alpha & \sin \alpha & 0 \\
-\sin \alpha & \cos \alpha & 0 \\
0 & 0 & 1
\end{array}\right]\left[\begin{array}{ccc}
1 & 0 & -a \\
0 & -1 & b \\
0 & 0 & 1
\end{array}\right]\left[\begin{array}{c}
x_{0} \\
y_{0} \\
1
\end{array}\right]
$$

According to formula(5), we can use this method which can correspond each point between the original figures and the new figures to complete the rotation operation about the picture.

Our method is based on this theory. Coordinate changed according to the angle of rotation when the image is rotated every times. Thus, it is very important to specify a fixed area to complete the image traversal. Also in this way, we can traverse the fixed area of the new image from the center to the edge of the torus in the every times that the image is rotated, and when the rotating angle attain(s) the 360 we will finish this task and make the change from the image to the histogram of 2D projection. As shown in Figure 5.

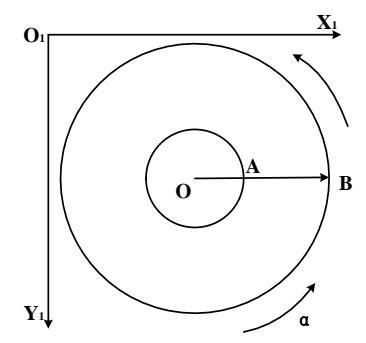

Fig.5 Schematic diagram of 360 degrees rotation projection

As show in the above figure, the $\mathrm{X}_{1} \mathrm{O}_{1} \mathrm{Y}_{1}$ coordinate system is used as a coordinate system which for traversing pixels. A fixed area which height is one pixel point and width is the same as the circle radius will be traversed pixels from A to B when the image is clockwise rotated a angle of $\alpha$. And we can extract the effective information because the most important information of the wheel is located in the plane of the circular ring from A to B. So our method can show the information that maximumly located in the effective area, and we also can segment the effective area from the whole image more convenient. Figure 6 is the impression drawing of pretreatment which is formed by the image of aluminum wheel rotate projects.
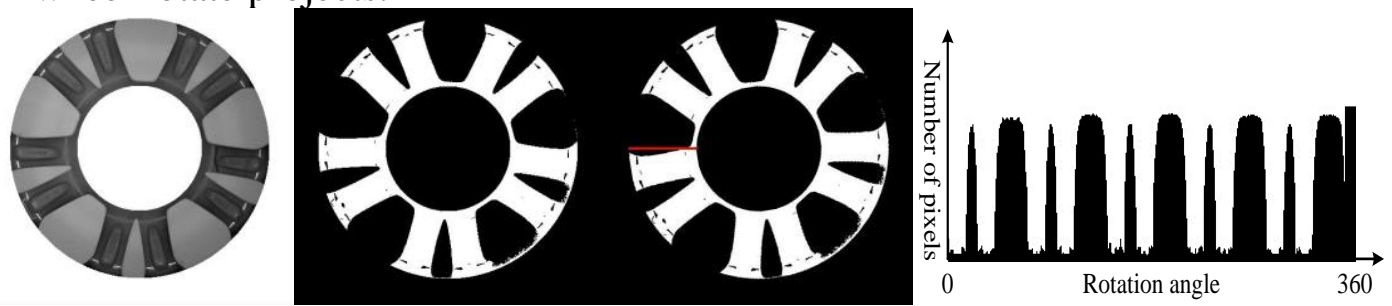

(a)Circular area image (b)Binarization (c)rotation projection

(d) Rotation histogram

Fig.6 Process diagram of 360 degree rotation projection

As shown above, Figure 6(c) is the rotation projection impression drawing when the image is rotated 10 degrees. The red line shown in the image is the location that the fixed area which need to be traversed. In this way, we can get the number of white pixel point through traversing the fixed area that red line is located to draw a histogram. Figure 6(d) is the projection histogram which is draw based on Figure 6(c), it can show the distribution of the number of pixels both black and white from the center to the edge about every angle.

\subsubsection{Image segmentation}

As shown in Figure 6(d), we can find the column that has the most white point by the method of mathematical statistics, and the column is the rotation angle. The projection histogram is based on black pixels as the bottom and use the white pixels point, which is counted by traversing pixels, to fill. So, the column which has more white pixels is the effective area that has useful information in the binaryzation image of aluminum wheel, and this part is need to be retained. Conversely, the column which has more black pixels is the blank area that should be removed. Thus, we can find the angle of the mean line of the effective area easily from histogram. But, some interference pixels appear at the bottom of the histogram. So, in order to segment the wheel image in a better way, we use the double 
threshold adaptive method to process the data of histogram. At first, we set the first threshold for the histogram and remove the interference pixels. Its formula is expressed by formula (6):

$$
f(x)=\left\{\begin{array}{cc}
100 & T>=30 \\
0 & T<30
\end{array}\right.
$$

As shown above, the $f(x)$ is number of the pixels of every column, the $x$ is horizontal coordinate value, the $T$ is first threshold. And the processed histogram is show in Figure 7.

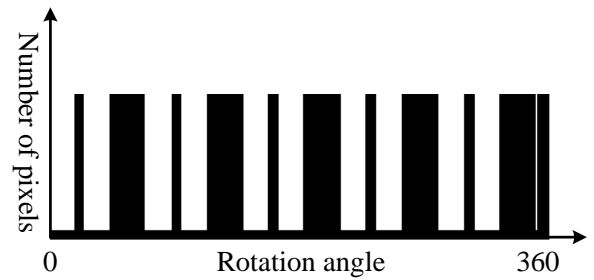

Fig.7 Processed histogram by first threshold

The second threshold is the segmentation threshold, this threshold is a adaptive value, it is expressed by formula (7) and formula (8):

$$
\begin{aligned}
& M_{i}=a_{x} \quad(f(x)=0) \\
& T_{1}=\frac{M_{i}+M_{i-1}}{2} \quad\left(M_{i}-M_{i-1}>0\right)
\end{aligned}
$$

The $M$ is a new sequence that used to storage the $a$ which is meet the conditions, the $a$ is rotation angle, the $T_{1}$ is second threshold. Next, we use the ROI method to segment the aluminum wheel under the condition of rotation when the rotation angle meets $T_{1}$. As shown in Figure 8.

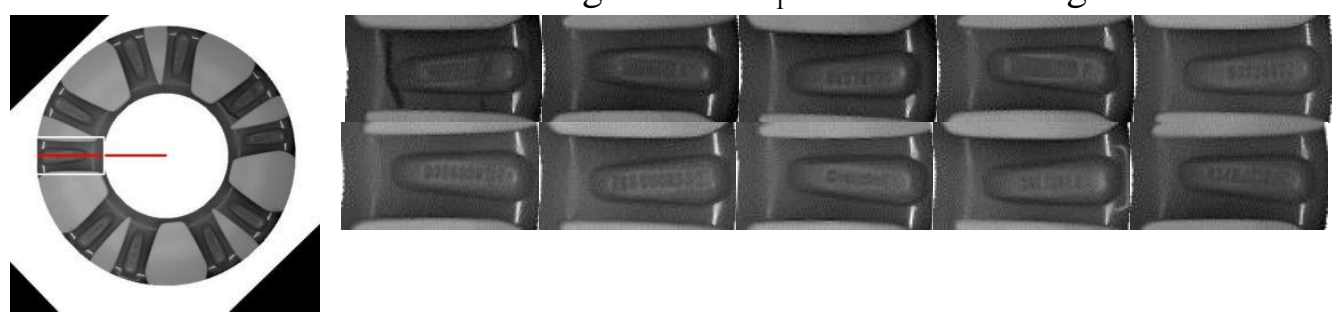

Fig.8 Impression drawing of segmentation of effective area

As shown in Figure 8, the red line is the center line of effective area, when it is arrived at the middle of the ROI that determined by histogram, it will be segment and extract at once, but the original image continues to rotate to find the next position which can meet the segmentation conditional. When the rotation angle arrived at 360 degrees, the task would have been done.

\section{Test verification}

This experiment collected 3 types of total graph of the back cavity of aluminum wheel, and collected 10 pictures every type to do the experiment in our method. By this way, we can validates the method's effectiveness what we have proposed in this paper. This experiment is the use of FJ series cameras, VS2010+Opencv2.4.9 software on the Window7 system using C++ language simulation experiment, as shown in Figure 9 for the re-acquisition of 3 types of map of the back cavity of aluminum wheel.

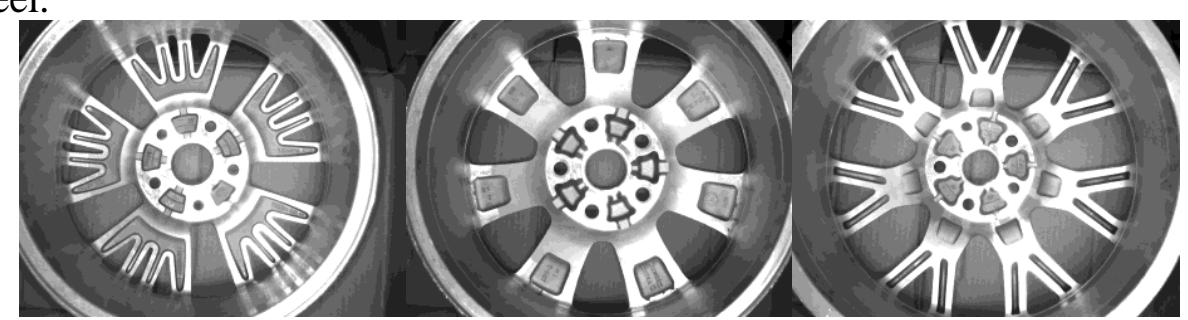

Fig.9 Original image of experimental wheel 


\subsection{Segmentation and extraction of effective region}

Using the re-acquisition picture of the 3 types of aluminum wheel to take a processing operation by the method what we have proposed in this paper. They are type I, type II and type III from left to right. The first, pretreatment reached preliminary extraction to get the ring part remains, where the main information of the back cavity, and then projection segmentation at the annular region, at last, an effective region is obtained which has the important information. The experimental process is shown in Figure 10:

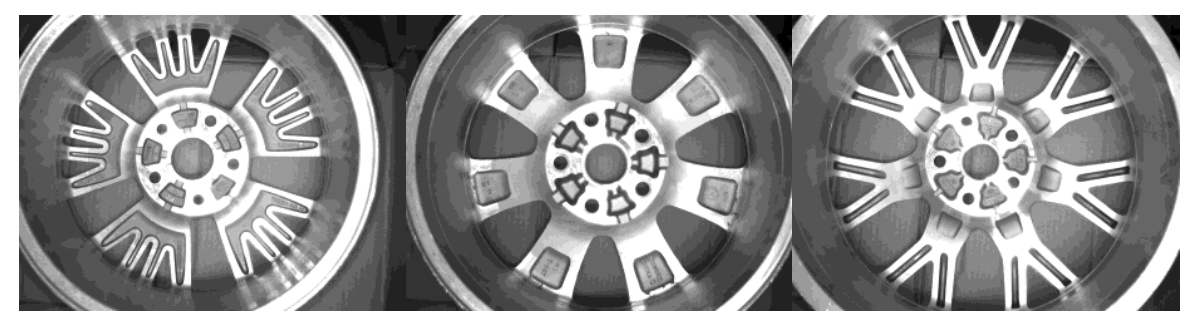

(a)Original image
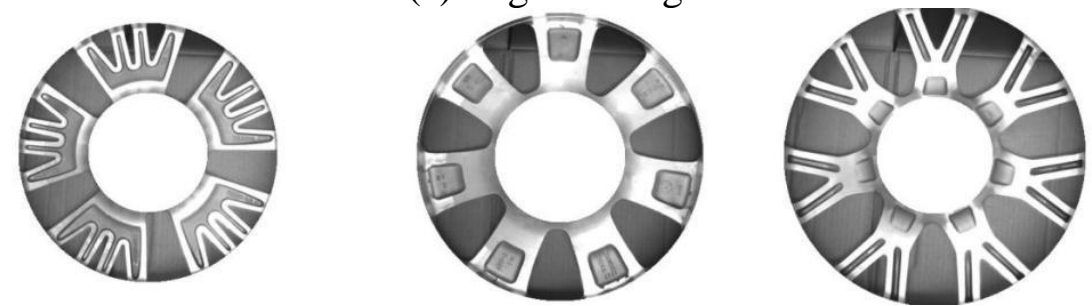

(b)Annular image

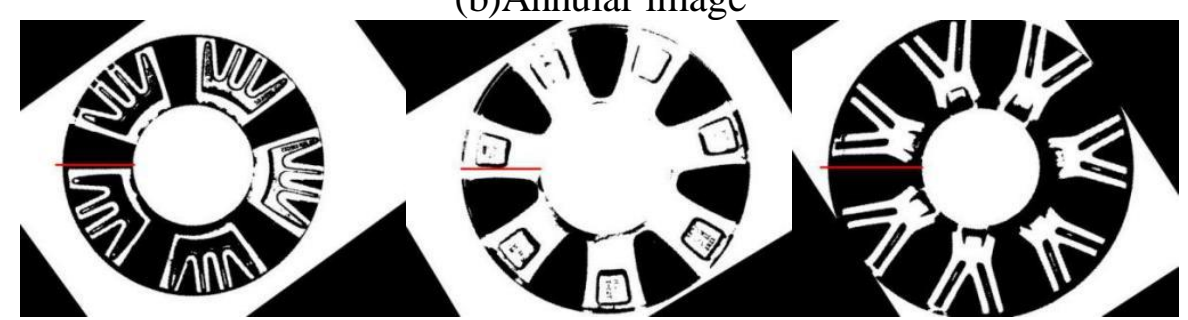

(c)The process image of rotating projection scanning

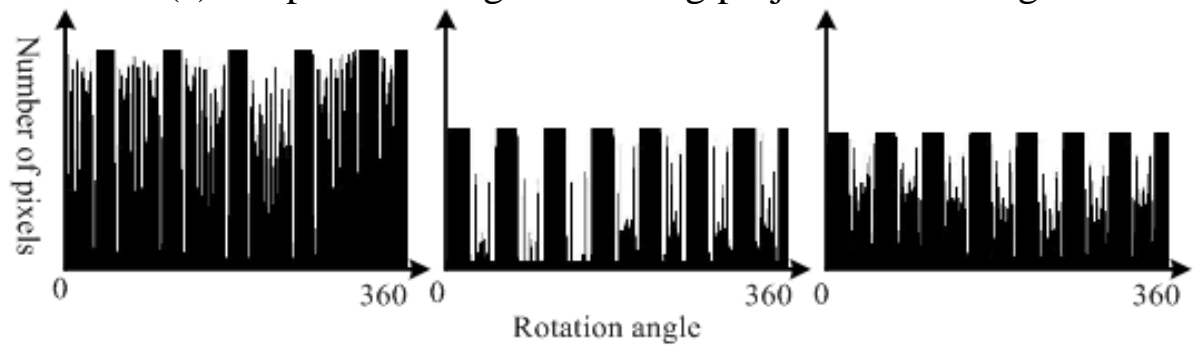

(d)Projection histogram

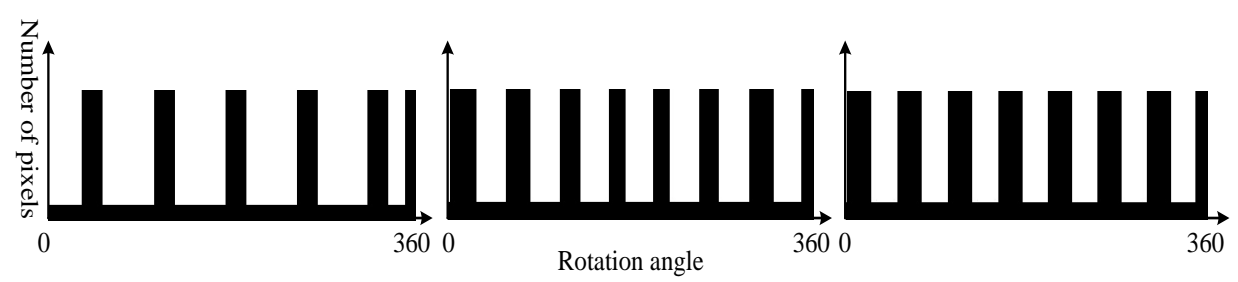

(e)Processed histogram by first threshold

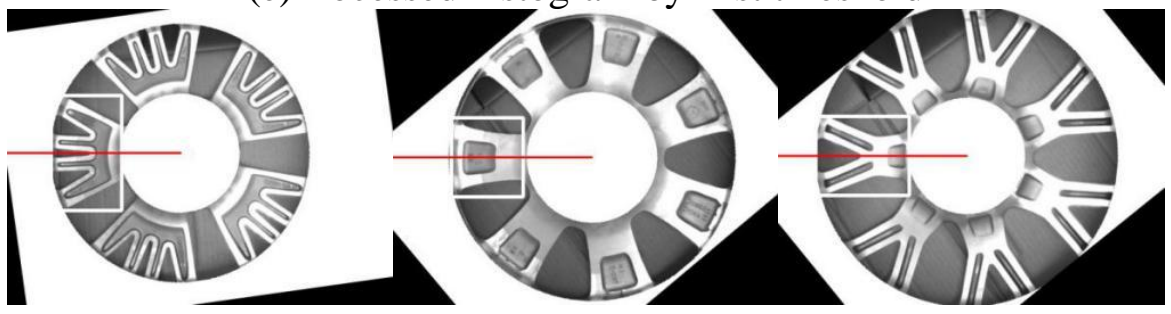

(f)Process image of segmentation and extraction 

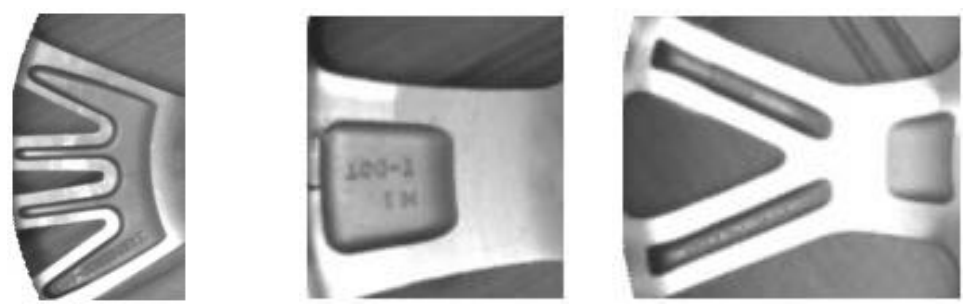

(g)Effective area image

Fig.10 Experimental process image

As shown above, We can clearly see that the method proposed in this paper can be a good way to extract the effective area where has the most important information on the aluminum wheel and prepare for the identification of regulation marking.

As shown in table 1, we count correct rate and time of segmentation and extraction of 30 images of the back cavity of aluminum wheel.

Table1 Correct rate and time of segmentation

\begin{tabular}{c|ccc}
\hline Wheel type & Type I & Type II & Type III \\
\hline $\begin{array}{c}\text { Porrect rate of } \\
\text { segmentation }\end{array}$ & $100 \%$ & $100 \%$ & $100 \%$ \\
$\begin{array}{c}\text { Segmentation and } \\
\text { extraction time(S) }\end{array}$ & 23.745 & 21.570 & 23.549 \\
\hline
\end{tabular}

From the table above we can see that the correct rate of segmentation is very high, but the segmentation and extraction time is too long. So, we should optimize this method in the next stage and the time limit in 15 seconds.

\section{Conclusion}

This paper mainly researches on the dynamic segmentation method of aluminum wheel images based on rotation histogram. This method combines the rotation projection method and dynamic extraction method, and they are succeed to complete the extraction of the effective area of the aluminum wheel. And through the re-acquisition pictures of 30 aluminum wheels to do the experiment. Verify the feasibility of this method in the field of dynamic segmentation of the aluminum wheel images. Besides, this article also has the place that need to be improved. The histogram' further accurate segmentation and illumination effects on the experiment caused a certain impact, because the wheel type I and III have narrow spokes, the method of extraction need to be improved to reduce its segmentation time. These are important contents that need to research in next stage.

\section{References}

[1]Ahmadvand,Ali, Kabiri,Peyman. Multispectral MRI image segmentation using Markov random field model[J]. Signal, Image and Video Processing. Vol.10 (2016) No. 2, p. 251-258.

[2]Gumus,Ergun. Segmentation and root localization for analysis of dental radiographs[J]. Signal, Image and Video Processing. Vol.10(2016)No.6, p.1073-1079.

[3]Peker,Murat, Karakaya,Fuat. SIH:segmented intensity histogram for orientation estimation in image matching[J]. Signal, Image and Video Processing. Vol.10(2016)No.6, p.1135-1142.

[4]Liu,L., Zhao,Z.M.. A novel image analysis method for rotational motion of circular feature based on perspective projection[J]. Imaging Science Journal. Vol.63(2015)No.5, p. 252-262.

[5]Wentzell,Peter D., Hou,Siyuan, Silva,Carolina Santos, et al. Procrustes rotation as a diagnostic tool for projection pursuit analysis[J]. Analytica Chimica Acta. Vol.887(2015),p. 51-63. 
[6]Wei,Bohong, Wang,Fan, Hu,Xiaopeng. Rotation-invariant Template Matching Based on Ring Projection and Orientation Codes[C]. 5th International Conference on Intelligent Control and Information Processing, ICICIP 2014. Dalian, Liaoning, China, August 18, 2014 - August 20, 2014, pp.192-197.

[7]Min, Y., Jiawei,L., Zhongchuan,L. Locating of $2 \pi$-projection view and projection denoising under fast continuous rotation scanning mode of micro-CT[J]. Neurocomputing. Vol.207(2016), p.335-345.

[8]Dong,Meng, Eramian,Mark G., Ludwig,Simone A., Pierson,Roger A.. Automatic detection and segmentation of bovine corpora lutea in ultrasonographic ovarian images using genetic programming and rotation invariant local binary patterns $[\mathrm{J}]$. Medical and Biological Engineering and Computing. Vol.51(2013)No.4, p.405-416.

[9]Lappan,Uwe, Wiesner,Brigitte, Scheler,Ulrich. Rotational Dynamics of Spin-Labeled Polyacid Chain Segments in Polyelectrolyte Complexes Studied by CW EPR Spectroscopy[J]. Macromolecules. Vol.48(2015)No.11, p.3577-3581.

[10]Duan,Lixiang, Yao,Mingchao, Wang,Jinjiang, et al. Segmented infrared image analysis for rotating machinery fault diagnosis[J]. Infrared Physics \& Technology. Vol.77(2016), p. 267-276.

[11]Lin,Long, Wang,Sihong, Xie,Yannan, et al. Segmentally Structured Disk Triboelectric Nanogenerator for Harvesting Rotational Mechanical Energy[J]. Nano Letters. Vol.13(2016)No.6, p. 2916-2923.

[12]Famin,Vincent, Raimbourg,Hugues, Garcia,Sebastian, et al. Stress rotations and the long-term weakness of the Median Tectonic Line and the Rokko-Awaji Segment[J]. Tectonics. Vol.33(2014)No.10, p.1900-1919.

[13]Gupta,Sayantan Datta, Rajamohan,Vasudevan. Segment optimization of a rotating multilayer sandwich beam[J]. Journal of Sandwich Structures and Materials. Vol.16(2016)No.2, p.148-172.

[14]Wachowski,Martin Michael, Wagner,Markus, Weiland,Jan, et al. Does total disc arthroplasty in $\mathrm{C} 3 / \mathrm{C} 4$-segments change the kinematic features of axial rotation. Journal of Biomechanics[J]. Vol.46(2013)No.10, p.1739-1745. 